\title{
Correction to: Electrogastrogram Based Medical Applications an Overview and Processing Frame Work
}

Ahmed Al Taee and Adel Al-Jumaily (D)

\section{Correction to:}

Chapter "Electrogastrogram Based Medical Applications an Overview and Processing Frame Work" in: A. M. Madureira et al. (Eds.): Hybrid Intelligent Systems, AISC 923, https://doi.org/10.1007/978-3-030-14347-3_50

The original version of the book was inadvertently published with the author's name as 'Ahmad A. Al-Tae' in chapter 50, which has now been changed to 'Ahmed Al Taee'. 